OPEN ACCESS

Edited by:

Chiara Briani,

University of Padua, Italy

Reviewed by:

Gianni Sorarù,

University of Padua, Italy

Maria Pennuto,

University of Padua, Italy

Haruhiko Banno,

Kyoto University, Japan

Carlo Rinaldi,

University of Oxford, United Kingdom

*Correspondence:

Viktorija Kenina

kenina.viktorija@gmail.com

†These authors have contributed equally to this work

Specialty section

This article was submitted to Neuromuscular Diseases,

a section of the journal

Frontiers in Neurology

Received: 23 July 2020

Accepted: 23 December 2020

Published: 20 January 2021

Citation:

Millere E, Rots D, Glazere I, Taurina G, Kurjane N, Priedite V, Gailite L,

Blennow $\mathrm{K}$, Zetterberg $\mathrm{H}$ and Kenina V (2021) Clinical Phenotyping and Biomarkers in Spinal and Bulbar Muscular Atrophy.

Front. Neurol. 11:586610.

doi: 10.3389/fneur.2020.586610

\section{Clinical Phenotyping and Biomarkers in Spinal and Bulbar Muscular Atrophy}

\author{
Elina Millere ${ }^{1,2,3+}$, Dmitrijs Rots ${ }^{3 \dagger}$, leva Glazere ${ }^{4,5}$, Gita Taurina ${ }^{6}$, Natalja Kurjane ${ }^{5,7}$, \\ Viktorija Priedite $^{8}$, Linda Gailite ${ }^{3}$, Kaj Blennow ${ }^{9,10}$, Henrik Zetterberg $^{9,10,11,12}$ and \\ Viktorija Kenina ${ }^{5,13 *}$
}

${ }^{1}$ Department of Neurology and Neurosurgery, Children's Clinical University Hospital, Riga, Latvia, ${ }^{2}$ Department of Doctoral Studies, Riga Stradins University, Riga, Latvia, ${ }^{3}$ Scientific Laboratory of Molecular Genetics, Riga Stradins University, Riga, Latvia, ${ }^{4}$ Department of Neurology, Pauls Stradins Clinical University Hospital, Riga, Latvia, ${ }^{5}$ Department of Biology and Microbiology, Riga Stradins University, Riga, Latvia, ${ }^{6}$ Department of Medical Genetics and Prenatal Diagnostics, Children's Clinical University Hospital, Riga, Latvia, ${ }^{7}$ Outpatient Service Centre, Pauls Stradins Clinical University Hospital, Riga, Latvia, ${ }^{8}$ BIOCON Medical Laboratory, LCC BIOCON, Riga, Latvia, ${ }^{9}$ Department of Psychiatry and Neurochemistry, Institute of Neuroscience and Physiology, the Sahlgrenska Academy at the University of Gothenburg. Mölndal, Sweden, ${ }^{10} \mathrm{Clinical}$ Neurochemistry Laboratory, Sahlgrenska University Hospital, Mölndal, Sweden, ${ }^{11}$ Department of Neurodegenerative Disease, University College London Queen Square Institute of Neurology, London, United Kingdom, ${ }^{12}$ UK Dementia Research Institute at University College London, London, United Kingdom, ${ }^{13}$ Rare Disease Centre, Riga East Clinical University Hospital, Riga, Latvia

Background: Spinal and bulbar muscular atrophy (SBMA) or Kennedy disease [OMIM: 313200] is a rare X-linked neuromuscular disease. Patients commonly present with muscle cramps, tremors, leg weakness, dysarthria and dysphagia.

Methods: We deeply phenotyped and evaluated the possible extent of affected systems in all patients with SBMA in Latvia $(n=5)$. In addition, neurophysiological studies and blood analyses were used to perform a molecular diagnosis and evaluate biochemical values. We analyzed neurofilament light (NfL) as a possible biomarker.

Results: Neurological examination revealed typical SBMA clinical manifestations; all patients had small or large nerve fiber neuropathy. Three of five patients had increased neurofilament light levels.

Conclusion: The study confirms the systemic involvement in patients suffering from SBMA. Increased NfL concentration was associated with either peripheral neuropathy or decreased body mass index. The complex phenotype of the disease should be kept in mind, as it could help to diagnose patients with SBMA.

Keywords: Kennedy disease, spinal and bulbar muscular atrophy, phenotype, clinical features, biomarker, neurofilament

\section{INTRODUCTION}

Spinal and bulbar muscular atrophy (SBMA), also known as Kennedy disease, is a rare X-linked neuromuscular disease (1). It is caused by a CAG repeat expansion resulting in $>35$ CAGs in the first exon of the androgen receptor (AR) gene (polyglutamine disease) (2). The mutant AR aggregates in the nucleus of a cell and results in direct toxicity to the cell (mostly, lower motor neurons and myofibrils), which clinically results in slowly progressive adult-onset lower motor neuron damage and primary myopathy early in the course of the disease $(3,4)$. Interestingly, 
myopathic changes in transgenic mouse models of SBMA were observed prior to any motor neuron degeneration, illustrating motor neuron involvement only in the later stages of SBMA (5-7). Additionally, the polyglutamine expansion causes loss of receptor activity and results in androgen insensitivity.

The reported prevalence of SBMA is highly variable. Recent data in Italy indicated a SBMA prevalence of $2.58 / 100,000$ in the male population (8), while it is higher in regions with a suggested founder effect, such as in the Vasa region of Finland where the estimated prevalence is $15 / 100,000$ male inhabitants $(8,9)$. The exact prevalence, however, is still unknown, because SBMA is thought to be underdiagnosed (10).

Patients with SBMA commonly present with slowly progressive muscle weakness as the main clinical feature, which typically starts in the lower limbs proximally and progresses during the course of the disease to also involve the bulbar and distal limb muscle groups $(2,3)$. Other symptoms such as cramps, myalgia and postural hand tremors are frequently reported long before the onset of muscle weakness. Lower motor neuron signs are persistent, including diminished or absent deep tendon reflexes and fasciculations in the limb muscles, tongue, and chin, as well as muscle atrophy $(2-4,11)$. The onset of the neurological symptoms is variable and ranges from 20 to 60 years, but SBMA typically manifests during the fourth or fifth decade of life. The CAG repeat size inversely correlates with the disease onset, with longer repeats associated with earlier onset but not with disease progression or severity (4). Additionally, androgen insensitivity leads to gynecomastia, reduced fertility, and erectile dysfunction $(2,12)$.

Recent studies and separate case series have found that SBMA might also affect other parts of the nervous system and can be associated with metabolic alterations. Additional neurological features include peripheral neuropathies and dysfunction of the autonomic system, while metabolic alterations include dysregulation of both glucose homeostasis (glucose intolerance and diabetes) and lipid metabolism (dyslipidaemia). These findings suggest that SBMA is a systemic disorder $(2,12)$.

Determining disease progression in clinical trials with limited time frame for a slowly progressing disease could be challenging. Several possible neuronal and muscle biomarkers of SBMA severity have been previously studied. Creatinine, instead of creatinine kinase, demonstrates capabilities as a biomarker by reflecting SBMA severity via muscle mass decrease $(13,14)$. Blood concentrations of the axonal damage marker neurofilament light (NfL) has been reported to be unchanged in patients with SBMA (14).

The involvement of various systems has been previously assessed separately, analyzing one or several systemic manifestations mostly in a limited number of patients with SBMA. Therefore, in this study, we deeply phenotyped all patients with SBMA in Latvia to evaluate the possible simultaneous extent of the involved systems previously reported or suggested to be affected in SBMA, as well as analyzed possible biomarkers of SBMA.

\section{MATERIALS AND METHODS}

\section{Study Participants}

All patients diagnosed with spinal and bulbar muscular atrophy in Latvia (five unrelated Caucasian male patients) were included in this study. For the comparison of plasma NfL levels, 21 healthy male controls without known neurological disease or neurologic symptoms were enrolled in this study as a control group.

\section{Clinical and Laboratory Examination}

Clinical characteristics, including neurological status testing, 6min walking testing, and hand grip testing, were evaluated by a certified neurologist at the time of enrolment into the study.

All patients underwent neurophysiological studies, including nerve conduction study (NCS), needle electromyography (EMG), and quantitative sensory testing (QST). For analysis of autonomic nervous system (ANS) function, heart rate variability (ANS Analysis Professional) and sympathetic skin response testing were performed. 2 days before the ANS tests, the patients stopped medication(s) that could influence the results.

Blood samples were used for investigating the following biochemical and hormonal profiles: creatinine, creatine phosphokinase, lipidogram, thyroid hormones (thyroid stimulating hormone and free thyroxine), testosterone, and glycated hemoglobin.

The NfL concentration in plasma was determined using the single molecule array (Simoa) NfL assay (Quanterix, Billerica, MA). For a quality control (QC) sample with a concentration of $15.8 \mathrm{pg} / \mathrm{mL}$, repeatability was $5.2 \%$ and intermediate precision was $5.2 \%$. For a QC sample with a concentration of $50 \mathrm{pg} / \mathrm{mL}$, repeatability was $2.7 \%$ and intermediate precision was $3.2 \%$. The lower limit of quantification was $1.9 \mathrm{pg} / \mathrm{mL}$. The samples were analyzed by board-certified laboratory technicians who were blinded to the clinical data. The levels with controls were compared using Mann-Whitney $U$-test.

\section{Molecular Analysis}

The CAG repeat analysis in the $A R$ gene was performed as previously described (15). All analyses were performed using capillary electrophoresis, and the results were confirmed by Sanger sequencing for two patients with the shortest CAG repeats using the BigDye Terminator Kit v.3.1 (Thermo Fisher Scientific, USA), according to adapted manufacturer's protocol. The number of CAG repeats for longer alleles were calculated based on fragment size.

\section{Ethics}

The study was approved by the Central Medical Ethics Committee of the Republic of Latvia (Nr.3/18-03-21). All individuals signed an informed consent form and allowed anonymised publication of their clinical information and photos.

\section{RESULTS}

The study included all patients with SBMA in Latvia $(n=5)$ that were 34-68 years old. Patient characteristics are shown in 
Table 1. The CAG repeat numbers were variable (range 4754). All patients had no positive family history for SBMA or other neuromuscular diseases. Two of the patients also had autoimmune thyroiditis, and one of the patients had primary arterial hypertension. No other comorbidities were reported during the study. For two of the patients, SBMA manifested during their thirties, although the earliest onset was at the age of six based on the patient's anamnesis data (the first complains was inability to perform the same sports activities as other children). Almost all patients indicated their initial symptoms as general fatigue and muscle cramps.

Clinical and neurological examination revealed limb weakness in all patients. Weakness was more prominent proximally in the lower extremities and asymmetric between the left and right side for almost all patients (Table 2). Patient 4, with the earliest manifestation of the disease, also had the most severe weakness in the extremities. Facial muscle weakness, diffuse fasciculations, absent tendon reflexes, and tongue atrophy were present in all subjects (Figure 1). Two patients had dysphagia and two had dysarthria. During examination, all patients had prominent hand tremor, and Patient 3 also presented with bilateral myotonia in the palms. Sensory assessment revealed glove and stockinglike sensory impairment as hyperesthesia for Patients 1 and 3 and hypoesthesia for Patient 5. Gynecomastia was observed in four out of five patients. Patients without gynecomastia had low body mass index (BMI), $18.7 \mathrm{~kg} / \mathrm{m}^{2}$. All patients noted sweating disturbances, but due to the small sample size and subjectivity of the complaints, it was questionable whether these

TABLE 1 | Patient characteristics.

\begin{tabular}{|c|c|c|c|c|c|}
\hline & Patient 1 & Patient 2 & Patient 3 & Patient 4 & Patient 5 \\
\hline $\begin{array}{l}\text { Age at examination / age } \\
\text { at onset* (years) }\end{array}$ & $38 / 33$ & $46 / 36$ & $35 / 14$ & $34 / 6$ & $68 / 45$ \\
\hline Family history & Negative & Negative & Negative & Negative & Negative \\
\hline Children & 1 daughter & 1 son & - & - & 2 sons \\
\hline Body Mass Index $\left(\mathrm{kg} / \mathrm{m}^{2}\right)$ & 30.4 & 25.4 & 22.5 & 18.7 & 24.6 \\
\hline
\end{tabular}

*Self-reported by patient.

TABLE 2 | Clinical features.

\begin{tabular}{|c|c|c|c|c|c|}
\hline & Patient 1 & Patient 2 & Patient 3 & Patient 4 & Patient 5 \\
\hline Initial symptoms & General fatigue & General fatigue & General fatigue & $\begin{array}{c}\text { General fatigue } \\
\text { Muscle cramps } \\
\text { Muscle pain }\end{array}$ & $\begin{array}{l}\text { Fatigue and } \\
\text { muscle pain in } \\
\text { lower limbs }\end{array}$ \\
\hline Gynecomastia & $\begin{array}{l}\text { Asymmetrical, } \\
\text { since } 18 \text { y.o. }\end{array}$ & $\begin{array}{l}\text { Asymmetrical, } \\
\text { since } 38 \text { y.o. }\end{array}$ & $\begin{array}{l}\text { Asymmetrical, } \\
\text { since } 14 \text { y.o. }\end{array}$ & - & $\begin{array}{c}\text { Symmetrical, since } \\
40 \text { y.o. }\end{array}$ \\
\hline Limb weakness & $\begin{array}{l}\text { Asymetric } \\
\text { tetraparesis }\end{array}$ & $\begin{array}{l}\text { Asymetric } \\
\text { tetraparesis }\end{array}$ & $\begin{array}{l}\text { Asymetric } \\
\text { tetraparesis }\end{array}$ & $\begin{array}{l}\text { Asymetric } \\
\text { tetraparesis }\end{array}$ & $\begin{array}{l}\text { Asymetric } \\
\text { tetraparesis }\end{array}$ \\
\hline Dysphagia & - & + & - & - & + \\
\hline $\begin{array}{l}\text { Tongue atrophy } \\
\text { (Figure 1) }\end{array}$ & + & + & + & + & + \\
\hline Tendon reflexes & Absent & Absent & Absent & Absent & Absent \\
\hline Fasciculations & Diffuse & Diffuse & Diffuse & Diffuse & Diffuse \\
\hline Muscle cramps & & & & Diffuse & Diffuse \\
\hline Tremor & + & + & + & + & + \\
\hline Sensory assessment & $\begin{array}{l}\text { Hyperesthesia } \\
\text { (glove and } \\
\text { stocking type) }\end{array}$ & $\mathrm{N}$ & $\begin{array}{l}\text { Hyperesthesia } \\
\text { (glove and } \\
\text { stocking type) }\end{array}$ & $\mathrm{N}$ & $\begin{array}{l}\text { Hypoesthesia } \\
\text { (glove and } \\
\text { stocking type) }\end{array}$ \\
\hline
\end{tabular}



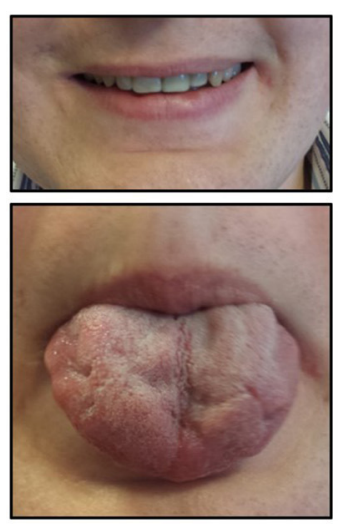

Patient 1
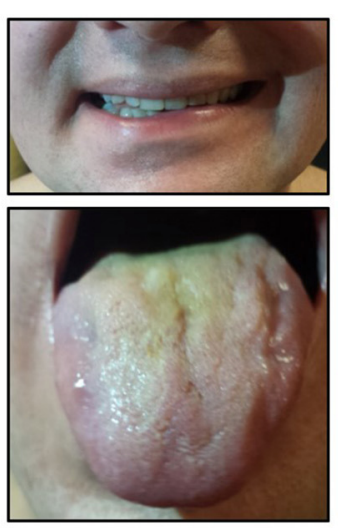

Patient 2
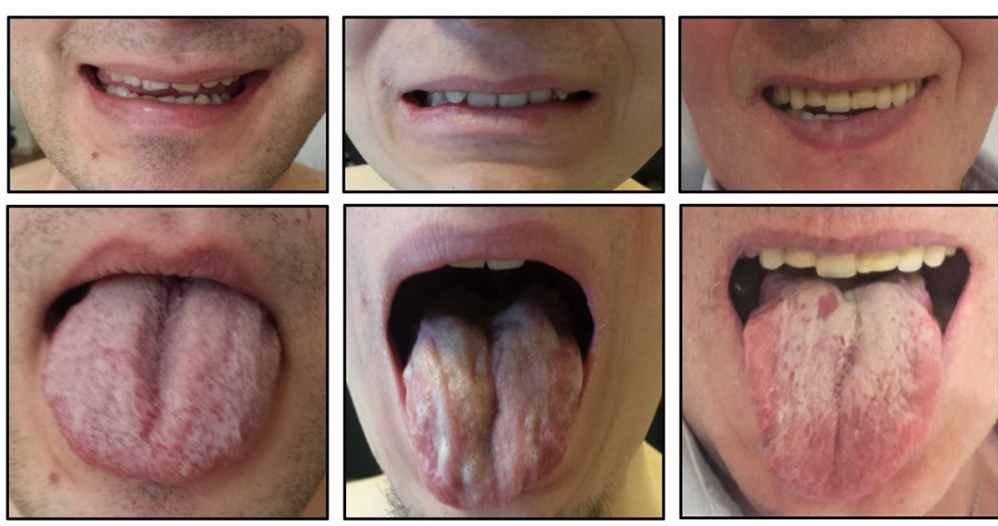

Patient 3

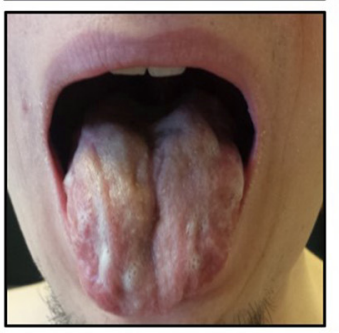

Patient 4

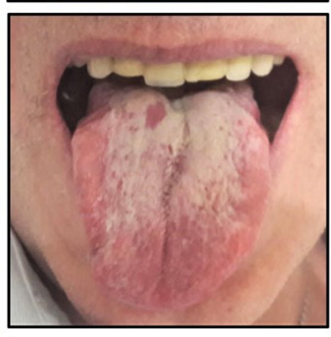

Patient 5

FIGURE 1 | Facial muscle weakness and tongue atrophy.

TABLE 3 | Patient functional characteristics.

\begin{tabular}{|c|c|c|c|c|c|}
\hline & Patient 1 & Patient 2 & Patient 3 & Patient 4 & Patient 5 \\
\hline $\begin{array}{l}\text { 6-min walking distance in meters } \\
\text { [norm value (16)] }\end{array}$ & $540(580)$ & $510(580)$ & $500(580)$ & 345 (580) & 195 (580) \\
\hline $\begin{array}{l}\text { Hand grip test right/left in kg } \\
\text { [age specific norm (17)] }\end{array}$ & $44 / 41(47 / 47)$ & $31 / 26(47 / 45)$ & $\begin{array}{l}30 / 30 \\
(47 / 47)\end{array}$ & $20 / 10(47 / 47)$ & $\begin{array}{l}25 / 20 \\
(40 / 38)\end{array}$ \\
\hline
\end{tabular}

TABLE 4 | Neurophysiological findings.

\begin{tabular}{|c|c|c|c|c|c|}
\hline & Patient 1 & Patient 2 & Patients 3 & Patient 4 & Patient 5 \\
\hline $\begin{array}{l}\text { Nerve conduction } \\
\text { studies }\end{array}$ & $\begin{array}{l}\text { Sensory demyelinating } \\
\text { polyneuropathy }\end{array}$ & Normal & Normal & Normal & $\begin{array}{l}\text { Severe motor, sensory } \\
\text { demyelinating axonal } \\
\text { polyneuropathy }\end{array}$ \\
\hline Electromyography & Neurogenic changes & Neurogenic changes & Neurogenic changes & Neurogenic changes & Neurogenic changes \\
\hline $\begin{array}{l}\text { Quantitative sensory } \\
\text { testing }\end{array}$ & $\begin{array}{l}\text { A delta type fiber } \\
\text { dysfunction }\end{array}$ & C type fiber dysfunction & C type fiber dysfunction & $\begin{array}{l}\text { A delta and C type fiber } \\
\text { dysfunction }\end{array}$ & $\begin{array}{l}\text { A delta and C type fiber } \\
\text { dysfunction }\end{array}$ \\
\hline $\begin{array}{l}\text { The heart rate variability } \\
\text { (ANS Analysis } \\
\text { Professional) }\end{array}$ & Normal & $\begin{array}{l}\text { Cardial autonomic } \\
\text { neuropathy } \\
\text { (tachycardia) }\end{array}$ & $\begin{array}{l}\text { Sympatotonuss } \\
\text { (tachycardia) }\end{array}$ & Parasympatotonuss & Normal \\
\hline $\begin{array}{l}\text { Sympathetic skin } \\
\text { response test }\end{array}$ & Normal & $\begin{array}{l}\text { Increased latency, } \\
\text { decreased amplitude }\end{array}$ & Increased latency & $\begin{array}{l}\text { Increased latency, } \\
\text { decreased amplitude }\end{array}$ & Normal \\
\hline
\end{tabular}

complaints could be associated with BMI. All patients had decreased 6-min walking distances (Table 3) (16). All patients had clearly decreased hand grip testing results with poor scores for both hands.

In all patients, needle EMG showed clear signs of neurogenic changes in all examined muscles, with large polyphasic motor unit potentials, fibrillation potentials, and fasciculations (Table 4). Motor and sensory nerve conduction velocities and compound muscle action potentials and sensory action potential amplitudes were abnormal for two out of five patients. Patient 1 had signs of sensory demyelinating polyneuropathy with decreased sensory conduction velocities, and Patient 5 had severe motor sensory demyelinating axonal polyneuropathy.
QST revealed abnormalities of tactile thresholds and mechanical pain perception in all patients, which was isolated in $\mathrm{C}$ sensory fiber types in two patients, A- $\delta$ fiber types in one patient and abnormalities in both types of sensory fibers in two patients.

Evaluation of the ANS via heart rate variability (ANS Analysis Professional) showed marked differences between patients. Patients 1 and 5 had no abnormalities; however, three patients had signs of ANS dysfunction. Patient 2 had signs of cardiac autonomic neuropathy (tachycardia), Patient 3 had sympathetic dysfunction, and Patient 4 had parasympathetic dysfunction. Sympathetic skin response testing results indicated that three out of five patients had peripheral sympathetic nervous system dysfunction, with all of them (3/5) demonstrating increased 
latency, and Patients 2 and 4 also had decreased amplitude during examination.

All patients had markedly elevated creatine phosphokinase levels, with the highest result reaching 5,000 U/L. All patient serum creatinine levels were within the normal range. To determine possible metabolic dysfunction linked to the pathogenesis of the disease, lipidogram, thyroid hormones, testosterone, and glycated hemoglobin levels were analyzed (Table 5). Two patients had increased testosterone levels, indicating androgen resistance. All patients had dyslipidaemia. Regarding thyroid function, two patients had autoimmune thyroiditis according to antibody (anti-thyroid peroxidase) and thyroid ultrasonography findings. Only Patient 3 had a subclinical decrease in thyroid function with increased thyroid stimulating hormone, despite demonstrating free thyroxine levels within the normal range.

NfL concentration was the highest for Patient $5(20.62 \mathrm{pg} / \mathrm{mL})$, followed by Patients $1(10.56 \mathrm{pg} / \mathrm{mL})$ and $4(10.01 \mathrm{pg} / \mathrm{mL})$. Our NfL control group consisted of 21 male participants, with a mean age of $27.3 \pm 11.7$ (range 5-55 years). There was no difference in age between the patient and control groups $(p>$ 0.05). The mean NfL chain concentration among control group participants was $5.08 \pm 1.76 \mathrm{pg} / \mathrm{mL}$, ranging from $2.60 \mathrm{pg} / \mathrm{mL}$ to $8.80 \mathrm{pg} / \mathrm{mL}$. Patient 5 had markedly increased NfL concentration in comparison with our control group, while Patients 1 and 4 also had higher NfL concentrations (>2.5 SD) than the control group. In total, SBMA patients group had higher NfL level than controls $(p<0.01)$.

\section{DISCUSSION}

In the current study, we have performed deep phenotyping and evaluation of possible biomarkers of all patients diagnosed with spinal and bulbar muscular atrophy in Latvia to unravel the extent of affected systems per patient, as well as to confirm that SBMA is a multisystemic condition.

Patients reported in this study exhibited typical features of SBMA with asymmetric atrophy and weakness of the proximal limb and bulbar muscles, as well as hand tremor consistent with lower motor neuron and skeletal muscle damage. Clinical, electrophysiological, and laboratory phenotyping of the patients allowed us to identify other symptoms and signs of SBMA that are rarely described or investigated. CAG repeat numbers in our SBMA group were variable (range 47-54). Although it has been reported that longer CAG repeats are associated with earlier onset of disease, our data did not show this association. However, our study group was very small. One reason for this could be that the onset of disease was reported by patients, while it could have started earlier or later than stated from the point of view of patients. CAG repeats are also associated with disease severity, which could have influenced our results. The mean CAG repeats in our group was 50.4, which was higher than previously reported (4).

Subclinical sensory involvement is common in patients with SBMA, affecting $70-100 \%$ of patients, even when motor nerve conduction studies are within reference range $(3,18)$. In SBMA, a typical feature is reduced sensory action potential amplitudes with axon loss, although decreased nerve conduction velocity and demyelination may also be present (19). In our study, all patients had small nerve fiber damage and two patients also had large nerve fiber damage detected in NCSs. Patient 1 had sensory demyelinating neuropathy, and Patient 5 had severe motor sensory demyelinating axonal polyneuropathy. There was no other confirmed cause for the severely manifested peripheral neuropathy. Sensory manifestations of SBMA in peripheral neuropathy and damage to both large and small nerve fibers are believed to be caused by the toxicity caused by accumulation of the mutant $\mathrm{AR}$ in dorsal root ganglion neurons in combination with metabolic alterations $(18,20,21)$. Small-fiber involvement in SBMA could explain the neuropathic pain reported in some patients (22). Antonini et al. previously evaluated small nerve fiber involvement in a cohort of six patients with SBMA and were not able to find damage to small myelinated $(A-\delta)$ and unmyelinated ( $\mathrm{C}$ types) nerve fibers by analyzing nerve biopsies of three patients with SBMA, although they found dysfunction in pain pathways among all six patients with SBMA that were

TABLE 5 | Biochemical and hormonal profile.

\begin{tabular}{|c|c|c|c|c|c|c|}
\hline & Normal range & Patient 1 & Patient 2 & Patient 3 & Patient 4 & Patient 5 \\
\hline Cholesterol (mmol/l) & $<5.0$ & 4.04 & 4.74 & 5.19 & 4.83 & 4.72 \\
\hline Triglycerides (mmol/l) & $<1.7$ & 2.71 & 1.12 & 1.48 & 0.98 & 2.36 \\
\hline Low density lipoprotein -cholesterol (mmol/l) & $<3.0$ & 2.19 & 3.06 & 3.4 & 2.82 & 2.38 \\
\hline Creatinine $(\mu \mathrm{mol} / \mathrm{L})$ & $76-90$ & 49 & 45 & 52 & 54 & 47 \\
\hline Free thyroxine (pmol/L) & $10.3-24.5$ & 16.4 & 14.9 & 14.05 & 14.3 & 10.18 \\
\hline Glycated Hemoglobin (\%) & $4.0-6.0$ & 4.9 & 5.5 & 4.9 & 4.7 & 5.3 \\
\hline Total testosterone level (ng/mL) & $3.30-8.05$ & 4.80 & 5.66 & 10.53 & 8.60 & 7.64 \\
\hline Neurofilament light (pg/mL) & $<9.5^{\star}$ & 10.56 & 4.37 & 5.20 & 10.01 & 20.62 \\
\hline
\end{tabular}

${ }^{*}$ Based on our control group data. 
investigated by using laser evoked potentials (23). However, another group was able to find the involvement of the small nerve fibers in two patients with SBMA by QST and skin biopsy analysis (20). Our data not only confirms the involvement of small nerve fibers in the pathogenesis of SBMA but also suggest that it may be a common feature among these patients.

The ANS is not considered to be affected during the course of SBMA, as patients usually do not exhibit symptoms suggesting ANS dysfunction. There is a study by Rocchi et al. analyzing ANS dysfunction in patients with SBMA, which analyzed five patients with SBMA without diabetes, impaired glucose tolerance, or cardiovascular diseases with five autonomic function tests (24). They observed subclinical dysfunction in the sympathetic and parasympathetic systems in four out of five patients (24). However, no patients exhibited symptoms of dysautonomia. Another group observed moderate autonomic skin denervation and reduced sweating in two patients with SBMA by analyzing skin biopsies and amount of sweating after pilocarpine stimulation (20). Our study confirms the involvement of the ANS and extends the knowledge of dysautonomic symptoms in patients with SBMA. Three out of five patients had involvement of the sympathetic or parasympathetic ANS, as shown by heart rate variability and sympathetic skin response test. It should be noted that two patients also demonstrated symptomatic ANS dysfunction. Patient 2 had tachycardia, and Patient 3 had tachycardia with increased sweating. Both patients were using beta-blockers to treat the tachycardia. Presentation of burning neuropathic pain in the distal extremities with dysautonomic symptoms, such as decreased sweating and difficulties with ejaculation, was recently reported in a patient with the highest number of CAG repeats known (68 CAG repeats) and confirms the involvement of small nerve fibers, as well as ANS, in the course of the disease (22). Previously reported damage to neurons from the AR toxicity in central neural autonomic regions (e.g., hypothalamus, nucleus ambiguous, and spinal intermediolateral nucleus, as well as in sympathetic ganglia) (21) could explain the involvement of the ANS among patients with SBMA. Additionally, it was suggested that ANS damage could also be caused by disruption of the small nerve fibers (post-ganglionic unmyelinated fibers) of the ANS (22). Altogether, our data, as well as previous data, show that the ANS is also involved in SBMAbut is rarely symptomatic. We find it peculiar that sensory polyneuropathy and ANS involvement were mutually exclusive. Polyneuropathy was reported in two patients, while ANS damage was reported in three patients. These findings are in agreement with the hypothesis that damage to different parts of the nervous system in SBMAmay have different pathogeneses.

Lower urinary tract symptoms (LUTS) without benign prostatic hyperplasia has been reported in up to $40 \%$ of the patients (25). The cause of LUTS in these patients is unknown and is hypothesized to be caused by androgen insensitivity. Despite the fact that none of our patients with dysautonomia reported LUTS, we hypothesize that at least some of the patients had LUTS as a result of ANS involvement (which was not recognized previously). It should be noted that LUTS could be present among our patient population but was not diagnosed, because it was not objectively evaluated using specific tools (e.g., International Prostate Symptom Score) in our patients.

Plasma NfL concentration is a known biomarker for various nervous system disorders in the central, as well as peripheral, nervous systems, reflecting axonal damage (26). A recent study investigated NfL as a biomarker of SBMA severity and found no difference in mean NfL levels between the SBMA patient and control groups, although several outliers with increased $\mathrm{NfL}$ levels were found (14). It should be noted that NfL level measurement was performed using the same method as in the previous study. In our study, three of five patients, however, demonstrated increased NfL levels. Two of these patients (Patients 1 and 5) had polyneuropathy confirmed by NCSs. Previous studies have shown that demyelinating and axonal forms of inherited neuropathies both have increased levels of NfL (27). We speculate that the NfL level for these two patients reflects peripheral nerve damage due to peripheral polyneuropathy. Patient 5 had severe polyneuropathy, as well as the highest NfL concentration. It has been reported that there is an age-dependent NfL increase, which could suggest that other factors were influencing the NfL level in Patient 5 (27). Patient 4 had increased NfL levels despite having no detectable polyneuropathy. Patient 4 had a BMI at the lower threshold of normal values $\left(18.7 \mathrm{~kg} / \mathrm{m}^{2}\right)$, which has been reported to result in a NfL increase in patients with anorexia nervosa (28). We hypothesize that increased NfL reflects the involvement of additional systems (peripheral nervous system) or other factors (age, BMI and other). The previous study by Lombardi et al. (14), which did not find NfL level differences between patients with SBMA and controls, did not describe whether patients with SBMA had involvement of additional systems. Therefore, we hypothesize that NfL can be used as a biomarker of peripheral polyneuropathy in SBMA but requires validation in a larger cohort with detailed evaluation of multisystemic involvement and other influencing factors, possibly in patients with SBMA from our geographic region.

All patients in our study had increased creatine phosphokinase levels, indicating muscle damage, and normal creatinine levels. Elevation of creatine phosphokinase in patients with SBMA could reflect myopathic damage. Creatinine for all our patient cohort was under reference range reflecting possible muscle damage (29). Laboratory studies confirmed a high prevalence of dyslipidaemia (reported in 4/5 patients) and a low prevalence of diabetes (no patient in our study group), as assessed by the glycated hemoglobin, which was similar to other studies $(2,18,30)$ although impaired glucose tolerance, which is common, was not assessed. Endocrinopathies (thyroid disorders or partial androgen insensitivity) is also common and affected all our patients. The metabolic abnormalities are believed to be caused by androgen insensitivity.

Heart muscle is usually spared despite the well-known primary myopathic damage in SBMA and AR accumulation that occurs in the cardiomyocyte nuclei $(18,31)$. Araki et al. described the common occurrence of Brugada syndrome in up $12 \%$ of patients with SBMA from Japan (31). Occurrence of Brugada syndrome was also observed among Caucasian 
patients with SBMA, although at a much lower frequency (up to $4 \%)(25)$. We were not able to find electrocardiographic abnormalities (excluding tachycardia in Patients 2 and 3), which was most probably due to the low frequency of cardiac involvement.

The main limitation of this study is the small sample size. However, we were able to analyse most of the symptoms reported in patients with SBMA, as well as replicate and confirm previous findings, to provide the information about the extent of affected systems by deeply phenotyping five patients with SBMA. Other features and symptoms reported in SBMA patients that were not analyzed in our study were only a subclinical change in brain metabolism, osteopenia, and non-alcoholic steatohepatitis $(18,32,33)$. Additionally, we were able to raise hypotheses that should be validated in larger cohorts. Besides the limited number of patients, another bias of our study is that our patients are younger than those analyzed in other studies, and the mean CAG repeat count was higher than in other studies. Unfortunately, muscle, skin or nerve biopsy was not available for further examination.

Our study confirms the systemic involvement in patients suffering from SBMA and expands knowledge about some of the features regarding ANS and small nerve fiber involvement, which have been inconclusive in previous studies. Our data show that small nerve fibers and the ANS are commonly affected in patients with SBMA. In our study, NfL showed biomarker capabilities with regard to the peripheral polyneuropathy in SBMA but requires validation in a bigger well-phenotyped cohort, where more factors influencing the NfL levels in patients are known. The complex phenotype of SBMA should be kept in mind, as it could help to identify and diagnose patients with SBMA, as well as provide explanations for the non-typical symptoms patients may have. In the age of therapy of genetic disorders, correct and timely diagnosis could also help in providing timely treatment for patients.

\section{REFERENCES}

1. Kennedy WR, Alter MSJ. Progressive proximal spinal and bulbar muscular atrophy of late onset: a sex-linked recessive trait. Neurology. (1968) 18:67180. doi: 10.1212/WNL.18.7.671

2. Querin G, Sorarù GP-FP. Kennedy disease (X-linked recessive bulbospinal neuronopathy): a comprehensive review from pathophysiology to therapy. Rev Neurol. (2017) 5204:243-360. doi: 10.1016/j.neurol.2017.03.019

3. Rhodes LE, Freeman BK, Auh S, Kokkinis AD, Pean AL, Chen C, et al. Clinical features of spinal and bulbar muscular atrophy. Brain. (2009) 132:324251. doi: 10.1093/brain/awp258

4. Fratta P, Nirmalananthan N, Masset L, Skorupinska I, Collins $\mathrm{T}$, Cortese A, et al. Correlation of clinical and molecular features in spinal bulbar muscular atrophy. Neurology. (2014) 82:2077-84. doi: 10.1212/WNL.0000000000000507

5. Gray AL, Annan L, Dick JRT, La Spada AR, Hanna MG, Greensmith L, et al. Deterioration of muscle force and contractile characteristics are early pathological events in spinal and bulbar muscular atrophy mice. Dis Model Mech. (2020) 13:dmm042424. doi: 10.1242/dmm.042424

6. Chivet M, Marchioretti C, Pirazzini M, Piol D, Scaramuzzino C, Polanco MJ, et al. Polyglutamine-expanded androgen receptor alteration of skeletal muscle homeostasis and myonuclear aggregation are affected by sex, age and muscle metabolism. Cells. (2020) 9:20325. doi: 10.3390/cells9020325

\section{DATA AVAILABILITY STATEMENT}

The original contributions presented in the study are included in the article/supplementary material, further inquiries can be directed to the corresponding author/s.

\section{ETHICS STATEMENT}

The studies involving human participants were reviewed and approved by the Central Medical Ethics Committee of the Republic of Latvia (Nr.3/18-03-21). The patients/participants provided their written informed consent to participate in this study. Written informed consent was obtained from the individual(s) for the publication of any potentially identifiable images or data included in this article.

\section{AUTHOR CONTRIBUTIONS}

VK, HZ, LG, and KB were involved in planning and supervised the study. EM, IG, and VK performed the clinical evaluation. DR, LG, and GT performed genetical analysis. EM, DR, and IG drafted the manuscript. NK, VP, VK, LG, GT, HZ, and KB made contribution to revising the paper and interpreting the results. All authors read and provided critical feedback on manuscript draft as well as approved the final version of manuscript.

\section{FUNDING}

HZ is a Wallenberg Scholar supported by grants from the Swedish Research Council (\#2018-02532), the European Research Council (\#681712), Swedish State Support for Clinical Research (\#ALFGBG-720931), the Alzheimer Drug Discovery Foundation (ADDF), USA (\#201809-2016862), the UK Dementia Research Institute at UCL, and Riga Stradins University Internal grant No. 131005.
7. Arnold FJ, Merry DE. Molecular mechanisms and therapeutics for SBMA/Kennedy's disease. Neurotherapeutics. (2019) 16:92847. doi: 10.1007/s13311-019-00790-9

8. Bertolin C, Querin G, Martinelli I, Pennuto M, Pegoraro E, Sorarù G. Insights into the genetic epidemiology of spinal and bulbar muscular atrophy: prevalence estimation and multiple founder haplotypes in the Veneto Italian region. Eur J Neurol. (2019) 26:519-24. doi: 10.1111/ene.13850

9. Udd B, Juvonen V, Hakamies L, Nieminen A, Wallgren-Pettersson C, Cederquist $\mathrm{K}$, et al. High prevalence of Kennedy's disease in western Finland: is the syndrome underdiagnosed? Acta Neurol Scand. (1998) 12833. doi: 10.1111/j.1600-0404.1998.tb01732.x

10. Arvin S. Analysis of inconsistencies in terminology of spinal and bulbar muscular atrophy and its effect on retrieval of research. J Med Libr Assoc. (2013) 101:147-50. doi: 10.3163/1536-5050.101.2.010

11. Grunseich C, Fischbeck K. Spinal and bulbar muscular atrophy. Neurol Clin. (2015) 33:847-54. doi: 10.1016/j.ncl.2015.07.002

12. Rosenbohm A, Hirsch S, Volk AE, Grehl T, Grosskreutz J, Hanisch F, et al. The metabolic and endocrine characteristics in spinal and bulbar muscular atrophy. J Neurol. (2018) 265:1026. doi: 10.1007/s00415-018-8790-2

13. Hijikata $\mathrm{Y}$, Hashizume $\mathrm{A}$, Yamada $\mathrm{S}$, Inagaki $\mathrm{T}$, Ito $\mathrm{D}$, Hirakawa $\mathrm{A}$, et al. Biomarker-based analysis of preclinical progression in spinal and bulbar muscular atrophy. Neurology. (2018) 90:e1501-9. doi: 10.1212/WNL.0000000000005360 
14. Lombardi V, Querin G, Ziff OJ, Zampedri L, Martinelli I, Heller C, et al. Muscle and not neuronal biomarkers correlate with severity in spinal and bulbar muscular atrophy. Neurology. (2019) 92:e1205 LP-11. doi: 10.1212/WNL.0000000000007097

15. Walsh S, Zmuda JM, Cauley JA, Shea PR, Metter EJ, Hurley BF, et al. Androgen receptor CAG repeat polymorphism is associated with fat-free mass in men. $J$ Appl Physiol. (2005) 98:132-7. doi: 10.1152/japplphysiol.00537.2004

16. The American Thoracic Society. ATS statement: guidelines for the six-minute walk test. Am J Respir Crit Care Med. (2002) 166:1117. doi: 10.1164/ajrccm.166.1.at1102

17. Massy-Westropp NM, Gill TK, Taylor AW, Bohannon RW, Hill CL. Hand Grip Strength: age and gender stratified normative data in a population-based study. BMC Res Notes. (2011) 4:127. doi: 10.1186/1756-05 00-4-127

18. Manzano R, Sorarú G, Grunseich C, Fratta P, Zuccaro E, Pennuto $\mathrm{M}$, et al. Beyond motor neurons: expanding the clinical spectrum in Kennedy's disease. Neurol Neurosurg Psychiatry. (2018) 89:808-12. doi: 10.1136/jnnp-2017-316961

19. Ni W, Chen S, Qiao K, Wang N, Wu Z-Y. Genotype-phenotype correlation in Chinese patients with spinal and bulbar muscular atrophy. PLoS ONE. (2015) 10:e0122279.doi: 10.1371/journal.pone.01 22279

20. Manganelli F, Iodice V, Provitera V, Pisciotta C, Nolano M, Perretti A, et al. Small-fiber involvement in spinobulbar muscular atrophy (Kennedy's disease). Muscle Nerve. (2007) 36:816-20. doi: 10.1002/mus.20872

21. Adachi H, Katsuno M, Minamiyama M, Waza M, Sang C, Nakagomi $\mathrm{Y}$, et al. Widespread nuclear and cytoplasmic accumulation of mutant androgen receptor in SBMA patients. Brain. (2005) 128:659-70. doi: 10.1093/brain/awh381

22. Grunseich C, Kats IR, Bott LC, Rinaldi C, Kokkinis A, Fox D, et al. Early onset and novel features in a spinal and bulbar muscular atrophy patient with a 68 CAG repeat. Neuromuscul Disord. (2014) 24:97881. doi: 10.1016/j.nmd.2014.06.441

23. Antonini G, Gragnani F, Romaniello A, Pennisi EM, Morino S, Ceschin V, et al. Sensory involvement in spinal-bulbar muscular atrophy (Kennedy's disease). Muscle Nerve. (2000) 23:2528. doi: 10.1002/(SICI)1097-4598(200002)23:2<252::AID-MUS17>3.0. CO;2-P

24. Rocchi C, Greco V, Urbani A, Di Giorgio A, Priori M, Massa R, et al. Subclinical autonomic dysfunction in spinobulbar muscular atrophy (Kennedy disease). Muscle Nerve. (2011) 44:737-40. doi: 10.1002/mus.22159

25. Querin G, Bertolin C, Da Re E, Volpe M, Zara G, Pegoraro E, et al. Nonneural phenotype of spinal and bulbar muscular atrophy: results from a large cohort of Italian patients. J Neurol Neurosurg Psychiatry. (2015) 87:8106. doi: 10.1136/jnnp-2015-311305

26. Kušnierová $\mathrm{P}$, Zeman $\mathrm{D}$, Hradílek $\mathrm{P}$, Cábal $\mathrm{M}$, Zapletalová $\mathrm{O}$. Neurofilament levels in patients with neurological diseases: a comparison of neurofilament light and heavy chain levels. J Clin Lab Anal. (2019) 33:e22948. doi: 10.1002/jcla.22948

27. Sandelius $\AA$, Zetterberg $H$, Blennow $K$, Adiutori R, Malaspina A, Laura $\mathrm{M}$, et al. Plasma neurofilament light chain concentration in the inherited peripheral neuropathies. Neurology. (2018) 90:e518-24. doi: 10.1212/WNL.0000000000004932

28. Nilsson IAK, Millischer V, Karrenbauer VD, Juréus A, Salehi AM, Norring C, et al. Plasma neurofilament light chain concentration is increased in anorexia nervosa. Transl Psychiatry. (2019) 9:180. doi: 10.1038/s41398-019-0518-2

29. Hannemann A, Friedrich N, Dittmann K, Spielhagen C, Wallaschofski H, Völzke H, et al. Age- and sex-specific reference limits for creatinine, cystatin $\mathrm{C}$ and the estimated glomerular filtration rate. Clin Chem Lab Med. (2011) 50:919-26. doi: 10.1515/cclm.2011.788

30. Nakatsuji H, Araki A, Hashizume A, Hijikata Y, Yamada S, Inagaki $\mathrm{T}$, et al. Correlation of insulin resistance and motor function in spinal and bulbar muscular atrophy. Neurology. (2017) 264:839-47. doi: 10.1007/s00415-017-8405-3

31. Araki A, Katsuno M, Suzuki K, Banno H, Suga N, Hashizume A, et al. Brugada syndrome in spinal and bulbar muscular atrophy. Neurology. (2014) 82:1813-21. doi: 10.1212/WNL.0000000000000434

32. Guber RD, Takyar V, Kokkinis A, Fox DA, Alao H, Kats I, et al. Non-alcoholic fatty liver disease in spinal and bulbar muscular atrophy. Neurology. (2017) 89:2481-90. doi: 10.1212/WNL.0000000000004748

33. Lai T-H, Liu R-S, Yang B-H, Wang P-S, Lin K-P, Lee Y-C, et al. Cerebral involvement in spinal and bulbar muscular atrophy (Kennedy's disease): a pilot study of PET. J Neurol Sci. (2013) 335:139-44. doi: 10.1016/j.jns.2013.09.016

Conflict of Interest: VP was employed by BIOCON Medical Laboratory, LCC BIOCON. HZ has served at scientific advisory boards for Denali, Roche Diagnostics, Wave, Samumed, Siemens Healthineers, Pinteon Therapeutics, and $\operatorname{CogRx}$, has given lectures in symposia sponsored by Fujirebio, Alzecure, and Biogen, and is a co-founder of Brain Biomarker Solutions in Gothenburg $\mathrm{AB}$ (BBS), which is a part of the GU Ventures Incubator Program (outside submitted work).

The remaining authors declare that the research was conducted in the absence of any commercial or financial relationships that could be construed as a potential conflict of interest.

Copyright (c) 2021 Millere, Rots, Glazere, Taurina, Kurjane, Priedite, Gailite, Blennow, Zetterberg and Kenina. This is an open-access article distributed under the terms of the Creative Commons Attribution License (CC BY). The use, distribution or reproduction in other forums is permitted, provided the original author(s) and the copyright owner(s) are credited and that the original publication in this journal is cited, in accordance with accepted academic practice. No use, distribution or reproduction is permitted which does not comply with these terms. 